\title{
UN climate change report turns up the heat
}

London. A report to the United Nations on the economic dimensions of climate change may be published with a key chapter missing unless a dispute can be settled between the chapter's authors and delegates from developing countries.

The chapter, which forms part of an overall study on the economic and social implications of climate change from working group three of the Intergovernmental Panel on Climate Change (IPCC), suggests that the costs required to slow down greenhouse gas emissions may exceed estimates of damage from climate change.

But the summary of the report, written explicitly for policy-makers and agreed by the working group in Montreal last month, effectively cancels this conclusion. Its writers argue that damage estimates would be higher if the chapter's authors had used the same criteria to assess losses in rich and poor countries, rather than, for example, estimating loss of life of an individual in a poor country at US $\$ 100,000$, one-fifteenth of the value in a rich country.

As a result, the authors of the chapter have decided to withhold their work unless they are allowed to respond to criticisms of their calculations made in the summary, which is written by experts from governments, particularly Cuba, India, Colombia and the Alliance of Small Island States.

"I would prefer to publish the chapter with an addendum making clear why we [the authors] disagree with the summary for policy-makers," says David Pearce, director of the Centre for Social and Economic Research of the Global Environment at University College London and the lead author for chapter six.

A senior IPCC official has said that any changes to the report's content should have been made during two earlier rounds of peer review among governments and independent experts. "At this stage, the authors can make a few editorial changes for clarity of reading, but not changes to the meaning or substance of the report," says James Bruce, co-chair of working group three.

Under IPCC rules, authors of chapters are responsible for overall editorial content. A chapter cannot be published by the panel unless all authors transfer their copyright to the climate body. One of the authors of chapter six, William R. Cline, a senior research fellow at the Institute for International Economics in Washington DC, agrees he would rather have the chapter erased from the IPCC's final report than see it included in its present form

But although Bruce concedes that the omission of the contentious chapter "could cause problems" for the IPCC, news of the chapter's probable withdrawal is likely to be welcomed in many developing countries, as well as in the London offices of the Global
Commons Institute (GCI), a group of environmentalists behind the campaign to "rewrite or withdraw" the chapter.

GCI has successfully lobbied developing countries to call for a recalculation of the damage that would result from a doubling of carbon dioxide concentrations by the year 2050 in a way that would require richer countries to shoulder more responsibility for the effects of climate change.

The chapter's authors had valued the damage from climate change at 1.5-2 per cent of gross world product (GWP), the

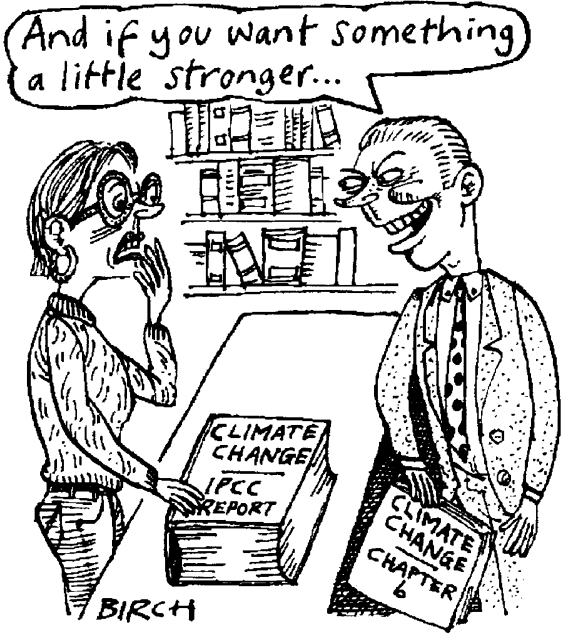

market value of all goods and services sold throughout the world. But GCI argues in a paper written for The Ecologist that damage estimates would be higher - between 12 per cent and 130 per cent of GWP - if based on a formula that asked countries how much compensation they would be willing to accept for the losses from climate change.

When the authors refused to alter their calculations, GCI persuaded those responsible for the summary for policy-makers to erase references to damage estimates, and include phrases such as "the literature on the subject in this section is controversial", and "the value of life" and "the loss of

unique cultures" cannot be quantified.

But Pearce argues that the GCI formula "is not supported by published data", and would not necessarily have increased the estimates for climate change damage in the developing world. One of the few known research papers to use the 'willingness to accept' method - from the Indira Gandhi Institute of Science in Bombay - resulted in an estimated $\$ 10,000$ value for loss of life.

Pearce claims that GCI has tried to turn an essentially scientific process into a political one. He says it should not have interfered with the process of independent scientific inquiry. "The IPCC is not a policymaking body. It is a body of scientific experts. We had strict instructions from the IPCC only to review the existing literature and not create any new literature."

But Aubrey Meyer, the director of GCI, disagrees and says Pearce and his team "are in no position" to label others with the charge of compromising scientific objectivity. Four out of the seven authors of the chapter, says Meyer, are the most frequently recurring names in the chapter's list of references. The same four names, he adds, form the majority of references linked to parts of the chapter dealing with damage estimates. "How can this be an objective process when the authors spent much of the time reviewing each other's work?" he asks.

Meyer says that the rule that authors cannot create literature should be lifted. $\mathrm{He}$ adds that the composition of authors should also better reflect the world's demography; only two of the chapter's seven authors were from the developing world.

But Pearce claims that Meyer and his colleagues have behaved "irresponsibly" in seeking to have quantitative references to damage estimates removed from the summary, as "99 per cent of all policy-makers will read this section, not the whole report".

The report is due to be approved at the IPCC's next plenary session in Rome next month. Ehsan Masood \& Ayala Ochert

\section{Re-engineering proposed for French agency}

Paris. Guy Aubert, director-general of the French Centre National de la Recherche Scientifique (CNRS), last week suggested that the agency's engineering sciences activities might be reorganized to improve their coordination with other disciplines and their ability to meet the needs of industry.

Speaking at a conference marking the twentieth anniversary of CNRS's engineering sciences department (SPI), Aubert proposed the setting up of a body to coordinate engineering across the agency. In particular, this body - known as an 'institute' in CNRS jargon - would oversee the growing number of collaboration projects between SPI and other CNRS laboratories in areas such as medicine, biology and sociology.

According to Aubert, the aim of the institute would be to reduce duplication and identify areas for cooperation. It would provide companies interested in working with CNRS with a single point of contact.

Aubert also proposed that a "national consortium of engineering sciences" should be set up as a "simple and informal" structure with similar aims to those of the proposed institute, but spanning all public research organizations, including the Atomic Energy Commission and the biomedical research agency INSERM. But he emphasized that both proposals were "only at the stage of intentions".

Declan Butler 